\title{
Monitoring the indirect impact of Covid-19 on hospital care pathways in Italy: results from the Mimico-19 network
}

Teresa Spadea ( $\square$ teresa.spadea@epi.piemonte.it)

ASL TO3 https://orcid.org/0000-0002-0771-0545

Chiara Di Girolamo

Regional Health and Social Services: Agenzia Sanitaria Regionale Emilia-Romagna

Tania Landriscina

ASL TO3

Olivia Leoni

Regional Epidemiological Observatory Lombardy Region

\section{Silvia Forni}

Regional Health Agency Tuscany Region

\section{Paola Colais}

Department of Epidemiology of the Regional Health Service Lazio: Dipartimento di Epidemiologia del Servizio Sanitario Regionale del Lazio

\section{Caterina Fanizza}

Regional Healthcare Agency Puglia Region: Regione Puglia

\section{Alessandra Allotta}

Epidemiological Observatory Sicily Region

Roberta Onorati

ASL TO3

Roberto Gnavi

ASL TO3

\section{Short Report}

Keywords: Covid-19, acute hospitalization, time-dependent care, elective surgery, Italy

Posted Date: July 8th, 2021

DOI: https://doi.org/10.21203/rs.3.rs-653542/v1

License: (c) (1) This work is licensed under a Creative Commons Attribution 4.0 International License. 

Monitoring the indirect impact of Covid-19 on hospital care pathways in Italy: results from the Mimico-19 network

Teresa Spadea, Epidemiology Unit ASL TO3, Piedmont Region, teresa.spadea@epi.piemonte.it

Chiara Di Girolamo, Regional Health and Social Care Agency of Emilia-Romagna Region, chiara.digirolamo@regione.emilia-romagna.it

Tania Landriscina, Epidemiology Unit ASL TO3, Piedmont Region, tania.landriscina@epi.piemonte.it

Olivia Leoni, Regional Epidemiological Observatory, Lombardy Region, olivia_leoni@regione.lombardia.it

Silvia Forni, Regional Health Agency of Tuscany Region, silvia.forni@ars.toscana.it

Paola Colais, Department of Epidemiology, Regional Health Service, Lazio Region, p.colais@deplazio.it

Caterina Fanizza, Regional Healthcare Agency of Puglia Region, c.fanizza@aress.regione.puglia.it

Alessandra Allotta, Department of Health Services and Epidemiological Observatory, Sicily Region, aleallotta@gmail.com

Roberta Onorati, Epidemiology Unit ASL TO3, Piedmont Region, roberta.onorati@epi.piemonte.it

Roberto Gnavi, Epidemiology Unit ASL TO3, Piedmont Region, roberto.gnavi@epi.piemonte.it and the Mimico-19 working group*

Corresponding author:

Teresa Spadea

Epidemiology Unit ASL TO3, Piedmont Region

Via Sabaudia 164, 10095 Grugliasco (Turin), Italy

Tel +3901140188334

email: teresa.spadea@epi.piemonte.it

ORCID iD - https://orcid.org/0000-0002-0771-0545

*The Mimico-19 working group:

Teresa Spadea, Roberto Gnavi, Tania Landriscina, Roberta Onorati, Alessandro Migliardi, Giuseppe Costa Epidemiology Unit, ASL TO3 Piedmont Region

Olivia Leoni, Michele Ercolanoni, Roberto Blaco - Regional Epidemiological Observatory, Lombardy Region

Chiara Di Girolamo, Elena Berti, Nicola Caranci, Maria Luisa Moro - Regional Health and Social Care Agency of Emilia-Romagna Region 
Silvia Forni, Valeria Di Fabrizio, Sara D'Arienzo, Fabrizio Gemmi, Mario Braga - Regional Health Agency of Tuscany Region

Paola Colais, Luigi Pinnarelli, Mariangela D'Ovidio, Maria Balducci, Marina Davoli - Department of Epidemiology, Regional Health Service, Lazio Region

Caterina Fanizza, Vito Petrarolo, Giulia Piepoli, Lucia Bisceglia - Regional Healthcare Agency of Puglia Region

Alessandra Allotta, Achille Cernigliaro, Salvatore Scondotto - Department of Health Services and Epidemiological Observatory, Sicily Region

Keywords: Covid-19; acute hospitalization; time-dependent care; elective surgery; Italy

\section{Declarations}

Funding: No funds, grants, or other support was received.

Conflicts of interest/Competing interests: The authors have no relevant financial or non-financial interests to disclose.

Availability of data and material: Individual anonymous data have been processed at each regional health department, under appropriate privacy regulations. Only unidentifiable aggregated data have been shared and will be available from the corresponding author upon reasonable request.

Code availability: Data cleaning and analyses were carried out using Excel and SAS standard commands and functions without generating any custom code.

Authors' contributions: Teresa Spadea, Chiara Di Girolamo and Roberto Gnavi contributed to the study conception and design. Material preparation, data collection and analysis were performed at local level by Roberta Onorati, Olivia Leoni, Chiara Di Girolamo, Silvia Forni, Paola Colais, Caterina Fanizza and Alessandra Allotta. Analysis and graphical presentation of the pooled indicators were performed by Tania Landriscina. The first draft of the manuscript was written by Teresa Spadea and Chiara Di Girolamo. All authors commented on previous versions of the manuscript, and read and approved the final manuscript. Additional declarations for articles in life science journals that report the results of studies involving humans and/or animals: Not applicable on unidentifiable aggregated data, derived from health information systems. 


\section{Abstract}

The COVID-19 pandemic has a non-negligible indirect impact on hospital care pathways, which is important to estimate. To this aim, we set up the Mimico-19 network of seven Italian regions (62\% of the Italian population) representing different socio-demographic areas of the country with also a different burden of the epidemic. We retrospectively analysed regional hospital discharges data, computing twelve indicators of volumes and performance in three clinical areas: cardiology, oncology and orthopaedics, including timedependent pathways and elective surgery. Weekly indicators for the period January-July 2020 were compared with the average of the corresponding indicators in 2018 and 2019; comparisons were performed within 3 sub-periods: pre-lockdown, lockdown and post-lockdown.

The weekly trend of hospitalizations for ST-segment elevation myocardial infarction (STEMI) showed a 40\% reduction, but the proportion of STEMI patients with a primary PTCA did not significantly change from previous years. Volumes of malignant neoplasms surgeries differed substantially by site, with a limited reduction for lung cancer (<20\%) and greater declines (30-40\%) for breast and prostate cancers. Hospitalizations for femoral neck fracture in the elderly decreased by $20 \%$, but the percentage of timely interventions remained constantly higher than the previous years. General trends did not show important differences across regions, regardless of the different Covid-19 burden.

Hospitalizations have generally decreased, but the capacity of a timely and effective response in timedependent pathways of care was not jeopardized throughout the period. The drop in the care demand for cardiovascular diseases and cancers needs to be further investigated and monitored more thoroughly. 


\section{Introduction}

The Covid-19 pandemic in Italy has caused more than 4,2 million cases of infection and over 130,000 deaths to date [1]. Following the first epidemic outbreak in Lombardy, which rapidly spread to other regions, the government issued the first national lockdown starting on 9 March 2020, and since then, evidence had accrued on its possible effects on people's health [2-3]. Services whose benefits could be lower than both the patient's risk of infection and the organizational difficulties were postponed, and citizens were recommended to avoid unnecessary access to health services. This indication, however, may have caused for fear of contagion or misinterpretation of the norm - further delays in recognizing symptoms and timely accessing diagnosis and treatment, even for non-deferrable conditions. Furthermore, several specialist departments had their hospital beds reduced to be used to face the huge flow of COVID-19 patients. Earlier in 2020, seven Italian regions (Piedmont, Lombardy, Emilia-Romagna, Tuscany, Lazio, Puglia and Sicily) have therefore set up the Mimico-19 network to monitor the side effects of the restrictive measures against Covid-19 on the quality of care. The seven regions total about 37 million inhabitants $(62 \%$ of the Italian population) and are representative of different geographical areas of the country, with a different epidemic burden [4].

The objective of this report is to describe the indirect impact of the pandemic and the lockdown on hospital activities through indicators of volume and performance in three clinical areas: cardiology, oncology, and orthopaedics, characterized by the high volumes of activity and the severity of the conditions.

\section{Methods}

Using data from the regional hospital discharge databases, we defined four indicators for each of the three clinical areas (the ICD-9-CM codes included in the indicators are detailed in the Online Resource 1):

- Cardiology: volumes of acute admissions for ST and non-ST segment elevation myocardial infarction (STEMI and NSTEMI); percentage of coronary angioplasty interventions (PTCA) in STEMI patients carried out within 90 minutes from the admission as an indicator of performance in a time-dependent procedure; in-hospital mortality in STEMI patients as an indicator of severity and outcome of the disease.

- Oncology: volumes of surgical interventions for all malignant neoplasms and those for lung, breast and prostatic cancers, representing a usually non-deferrable surgery, a proxy indicator of screening activities, and a scheduled operation, respectively. 
- Orthopaedics: volumes of hip and knee replacement, mostly scheduled surgery; acute admissions for femoral neck fracture in the elderly ( $>65$ years of age) and percentage of interventions in these patients performed within two days, as a second time-dependent procedure.

Regional indicators were pooled into national estimates. They were computed on a weekly basis for the period January-July 2020 and compared through the non-parametric Kruskall-Wallis test with the average of the corresponding months in 2018-2019, considering three sub-periods: pre-lockdown (until March 8), lockdown (March 9-May 17) and post-lockdown (from May 18 onwards). We also calculated the weekly percentage variation of the 2020 value vs. the $2018-19$ average.

\section{Results}

Here we report the results for the pooled indicators, highlighting only important regional variations (detailed regional results are presented in the Online Resource 2).

\section{Cardiology}

The weekly trend of hospitalizations for STEMI (Figure 1a) exhibited a gradual reduction in volumes, which began in late February and peaked in mid-April (\% variation about $-40 \%)$. Trends revealed a highly significant difference during lockdown, although STEMI hospitalization showed a significant $4 \%$ reduction even before the pandemic. In the post-lockdown, volumes went slowly back to the starting values. The hospitalization for NSTEMI (Figure 1b) showed a similar trend, but the average percent reduction was greater (over $55 \%$ at the end of March) and the post-lockdown recovery was slower. The proportion of STEMI patients with a primary PTCA (Figure 1c), which could be estimated only in four regions, remained stable over time without significant changes from previous years. On the other hand, in-hospital mortality significantly increased by $26 \%$ on average during lockdown and by $15 \%$ in the subsequent period (Figure 1d). This unfavourable outcome was almost entirely driven by Lombardy, the region with the earliest and greatest burden of Covid-19; all the other regions showed non-significant differences in mortality, although with large weekly fluctuations (Figure S1d, Online Resource 2).

Oncology

The total volume of malignant cancers surgeries (Figure 2a) showed a decline, which was moderate during the first two weeks of lockdown, reached $-25 \%$ by the end of March, and remained at these levels even in the post-lockdown. Trends differed by cancer site: the reduction in volumes was limited (less than $20 \%$ ) for lung cancers (Figure 2b), whereas it fell by about 30-40\% for breast and prostate surgery (Figures 2c-d), earlier for the latter and in the post-lockdown for breast cancer. 


\section{Orthopaedics}

Hospitalizations for femoral neck fracture in the elderly (Figure 3a) decreased by about $20 \%$ during lockdown, remaining significantly below the volumes of the previous two years in the post-lockdown too. In contrast, the percentage of timely interventions (Figure $3 \mathrm{~b}$ ) was constantly higher than the previous years throughout the whole period, with the difference increasing in the post-lockdown. Hip and knee replacements plunged dramatically following national indication to suspend scheduled operations. In both cases, however, there was an inversion of the trends by the end of July 2020 , with volumes significantly exceeding those in 2018-2019

\section{Discussion}

In summary, hospitalizations have generally decreased for most diseases and surgical interventions, but the changes in hospital supply and organization have not jeopardized the capacity of a timely and effective response in time-dependent pathways of care throughout the period. However, volumes of both acute hospitalizations and elective surgery have not returned to the starting levels in the post-lockdown months, even for potentially severe conditions. General trends did not show important differences across regions, regardless of the different burden of Covid-19, except for in-hospital mortality in STEMI patients in Lombardy.

The reduction in STEMI hospitalizations may be linked to a real lower incidence of heart attacks (e.g., related to better air quality or reduced mobility), but we cannot exclude delays in diagnosis or self-limitation of the demand by patients for fear of contagion. The secular trend of reduced hospitalization for STEMI may have also played a role, though marginal. Indeed, the lack of timely access to hospital care would also be supported by the reported increase in the number of out-of-hospital cardiac arrest deaths $[5,6]$. Moreover, we observed an increase in STEMI in-hospital mortality in Lombardy, where the high prevalence of Covid-19 was associated with worse STEMI outcomes [7]. This could also be associated with the delay in seeking hospital care by patients suffering a heart attack, which would result in a reduction in the efficacy of a primary PTCA.

The overall volume of cancer surgery hides profound differences across sites: in each of them, in fact, the decision to postpone the surgery depends on the balance between the benefit of an immediate operation and the risk of hospital infection from Covid-19 [8]. As expected, the decline in volumes of lung surgery was negligible, mainly due to its non-deferrable nature, while reductions for breast and prostate surgery were greater. Notably, especially for breast cancer, the persistence of the reduction in the post-lockdown is likely 
an indirect consequence of the suspension of screening activities [9], whose real impact on women's health will only be visible in the future. Indeed, a four-week delay in breast cancer surgery has been estimated to be associated with an $8 \%$ increased risk of mortality [10].

Hospitalizations for femoral neck fracture in the elderly decreased, probably due to the limited mobility imposed by the confinement measures, while hip and knee replacements were affected by the interruption of non-urgent surgery and the need to adapt surgical and outpatient activities to the new safety regulations [3]. This suspension has likely left room for an improvement in the timeliness management of emergencies, as in the case of the femoral neck fracture. Interestingly, the offer of orthopaedic surgery during the 2020 summer exceeded the volume of operations usually performed in this period. This reflects the timely and effective reorganisation of the sector after the lockdown in order to recover the accrued delays.

\section{Conclusion}

Rescheduling less urgent interventions may not affect survival in the short term but may have amplified the severity of unresolved health problems. Furthermore, the deferral involves a lengthening of waiting lists that will require a re-scheduling attentive to priorities, equity and efficiency. At this stage of the epidemic, it is necessary to strengthen primary care services so that they can adopt a pro-active approach and move towards the identification of risk conditions that were neglected during the pandemic and timely address patients to the secondary care system.

Finally, the drop in cardiovascular or oncological care needs to be monitored more thoroughly to counteract a possible reduced access to early diagnosis (as in the case of breast cancer screening) and follow-up of severe chronic diseases. 


\section{References}

1. Istituto Superiore di Sanità. COVID-19 integrated surveillance data in Italy. https://www.epicentro.iss.it/en/coronavirus/sars-cov-2-dashboard Accessed 16 Jun 2021.

2. De Rosa S, Spaccarotella C, Basso C, et al. on behalf of Società Italiana di Cardiologia and the CCU Academy investigators group. Reduction of hospitalizations for myocardial infarction in Italy in the COVID-19 era. Eu Heart J 2020; 41: 2083-2088. https://doi.org/10.1093/eurheartj/ehaa409.

3. Di Marzo F, Gemmi F, Cennamo R, et al. Impact of SARS-CoV-2 on elective surgical volume in Tuscany: effects on local planning and resource prioritization. Br J Surgery 2020; 107: e391-e392. https://doi.org/10.1002/bjs.11832.

4. Fabiani M, Onder G, Boros S, et al. Il case fatality rate dellinfezione SARS-CoV-2 a livello regionale e attraverso le differenti fasi dell'epidemia in Italia [The case fatality rate of SARS-CoV-2 infection at regional level, through the different epidemic phases in Italy]. Rapporto ISS COVID-19 n. 1/2021, Roma. https://www.iss.it/documents/20126/0/Rapporto+ISS+COVID-19+n.+1 2021.pdf/eef324b0-983d-c25796fd-e8d430e1ca82?t=1612179039051 Accessed 17 May 2021).

5. Baldi E, Primi R, Mare C, and All the Lombardia CARe researcher. Out-of-hospital cardiac arrest incidence in the different phases of COVID-19 outbreak. Resuscitation 2021; 159: 115-116. http://dx.doi.org/10.1016/j.resuscitation.2020.12.020.

6. Campo G, Fortuna D, Berti E, et al. In- and out-of-hospital mortality for myocardial infarction during the first wave of the COVID-19 pandemic in Emilia-Romagna, Italy: A population-based observational study. The Lancet Regional Health - Europe 2021. https://doi.org/10.1016/j.lanepe.2021.100055.

7. Carugo S, Ferlini M, Castini D et al. Management of acute coronary syndromes during the COVID-19 outbreak in Lombardy: The "macro-hub" experience. IJC Heart \& Vasculature 2020; 31. https://doi.org/10.1016/j.ijcha.2020.100662.

8. Hartman HE, Sun Y, Devasia TP, et al. Integrated survival estimates for cancer treatment delay among adults with cancer during the COVID-19 pandemic. JAMA Oncol. Published online October 29, 2020. doi:10.1001/jamaoncol.2020.5403.

9. Mantellini P, Battisti F, Armaroli P, et al. Ritardi maturati dai programmi di screening oncologici ai tempi del COVID-19 in Italia, velocità della ripartenza e stima dei possibili ritardi diagnostici [Oncological organized screening programmes in the COVID-19 era: an Italian survey on accrued delays, reboot velocity, and diagnostic delay estimates]. Epidemiol Prev 2020; 44 (5-6) Suppl 2:344-352. doi: 10.19191/EP20.5-6.S2.136. 
10. Hanna TP, King WD, Thibodeau S, et al. Mortality due to cancer treatment delay: systematic review and meta-analysis. BMJ 2020; 371:m4087. http://dx.doi.org/10.1136/bmj.m4087. 
Fig. 1 Impact of Covid-19 on cardiological hospital care in Italy: volumes and performance by sub-period* Weekly trend of indicators (left axis) with $95 \% \mathrm{Cl}$ and \% variations (right axis) - January-July 2020 vs. 201819 average

* pre-lockdown, lockdown (9/3-17/5), post-lockdown - p-values from the Kruskall-Wallis test for comparisons within each subperiod

Fig.2 Impact of Covid-19 on oncological hospital care in Italy: surgery volumes by sub-period*

Weekly trend of indicators (left axis) with $95 \% \mathrm{Cl}$ and \% variations (right axis) - January-July 2020 vs. 201819 average

* pre-lockdown, lockdown (9/3-17/5), post-lockdown - p-values from the Kruskall-Wallis test for comparisons within each subperiod

Fig.3 Impact of Covid-19 on orthopaedic hospital care in Italy: volumes and performance by sub-period* Weekly trend of indicators (left axis) with $95 \% \mathrm{Cl}$ and \% variations (right axis) - January-July 2020 vs. 201819 average

* pre-lockdown, lockdown (9/3-17/5), post-lockdown - p-values from the Kruskall-Wallis test for comparisons within each subperiod

Online Resource 1: List of indicators with their corresponding ICD-9-CM codes and selection criteria

Online Resource 2: Figures S1-S3 with detailed regional indicators 


\section{Figures}

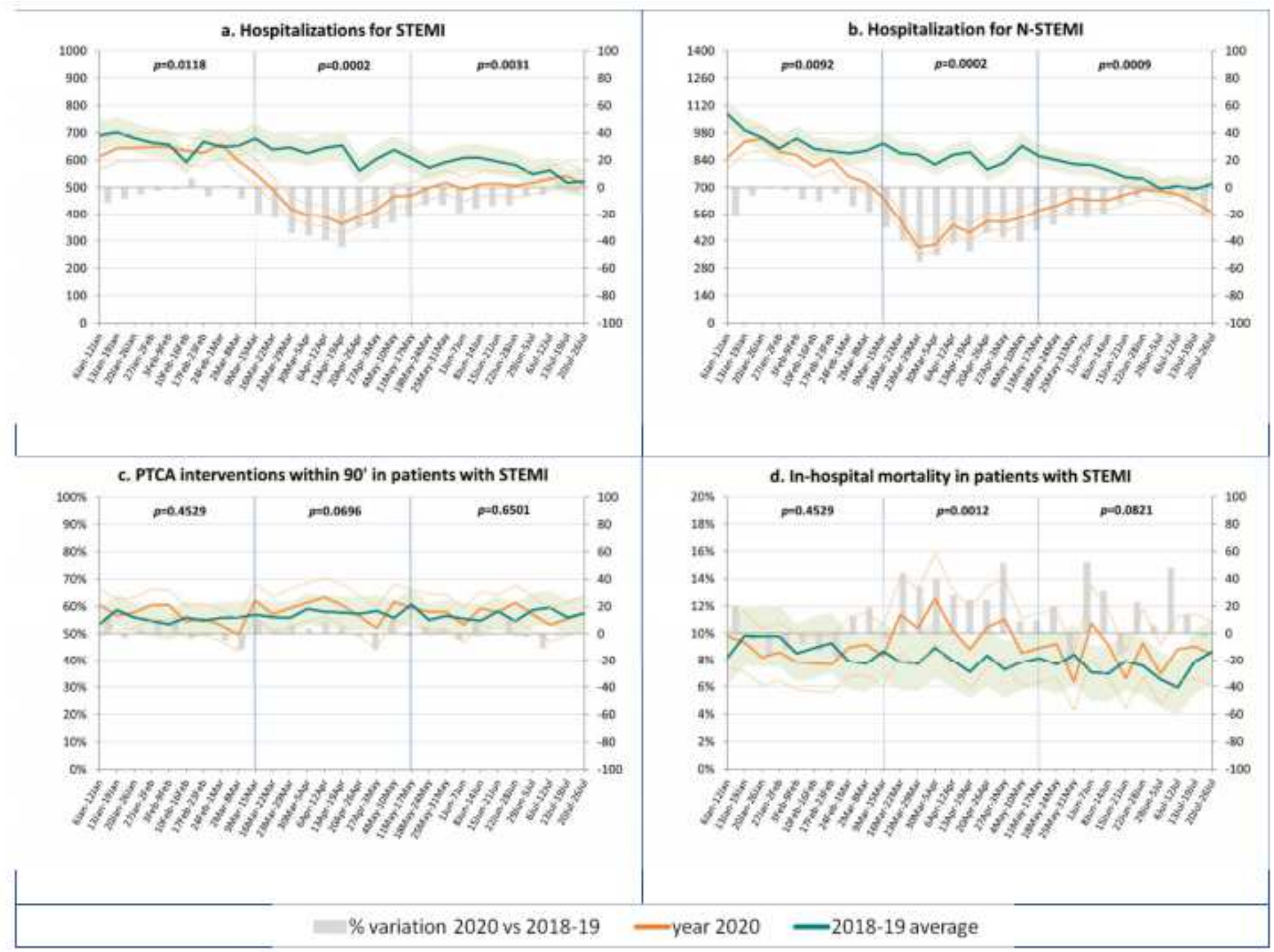

\section{Figure 1}

Impact of Covid-19 on cardiological hospital care in Italy: volumes and performance by sub-period* Weekly trend of indicators (left axis) with $95 \% \mathrm{Cl}$ and \% variations (right axis) - January-July 2020 vs. 2018-19 average. * pre-lockdown, lockdown (9/3-17/5), post-lockdown - p-values from the KruskallWallis test for comparisons within each subperiod 


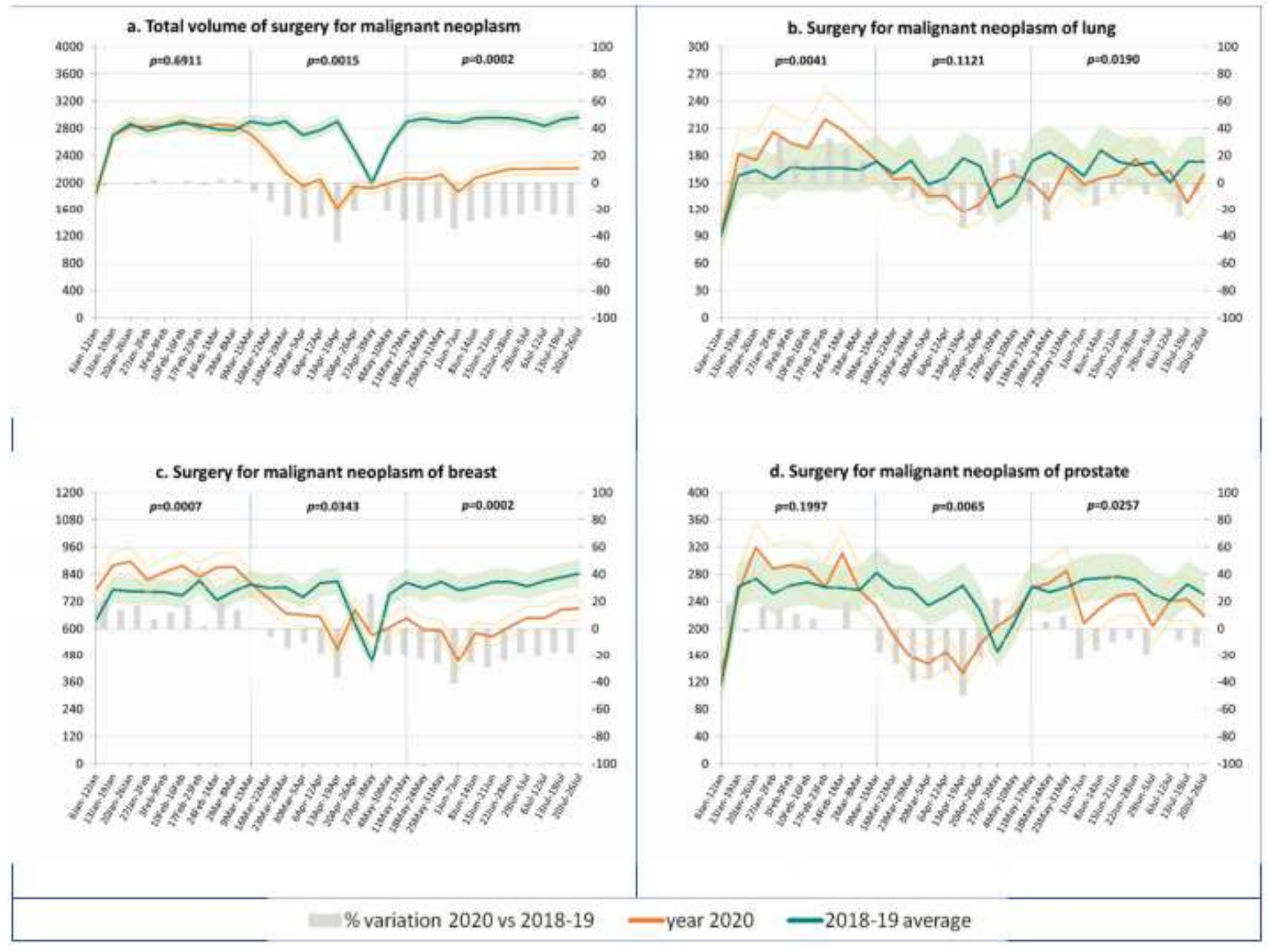

Figure 2

Impact of Covid-19 on oncological hospital care in Italy: surgery volumes by sub-period* Weekly trend of indicators (left axis) with $95 \% \mathrm{Cl}$ and \% variations (right axis) - January-July 2020 vs. 2018-19 average. * pre-lockdown, lockdown (9/3-17/5), post-lockdown - p-values from the Kruskall-Wallis test for comparisons within each subperiod 


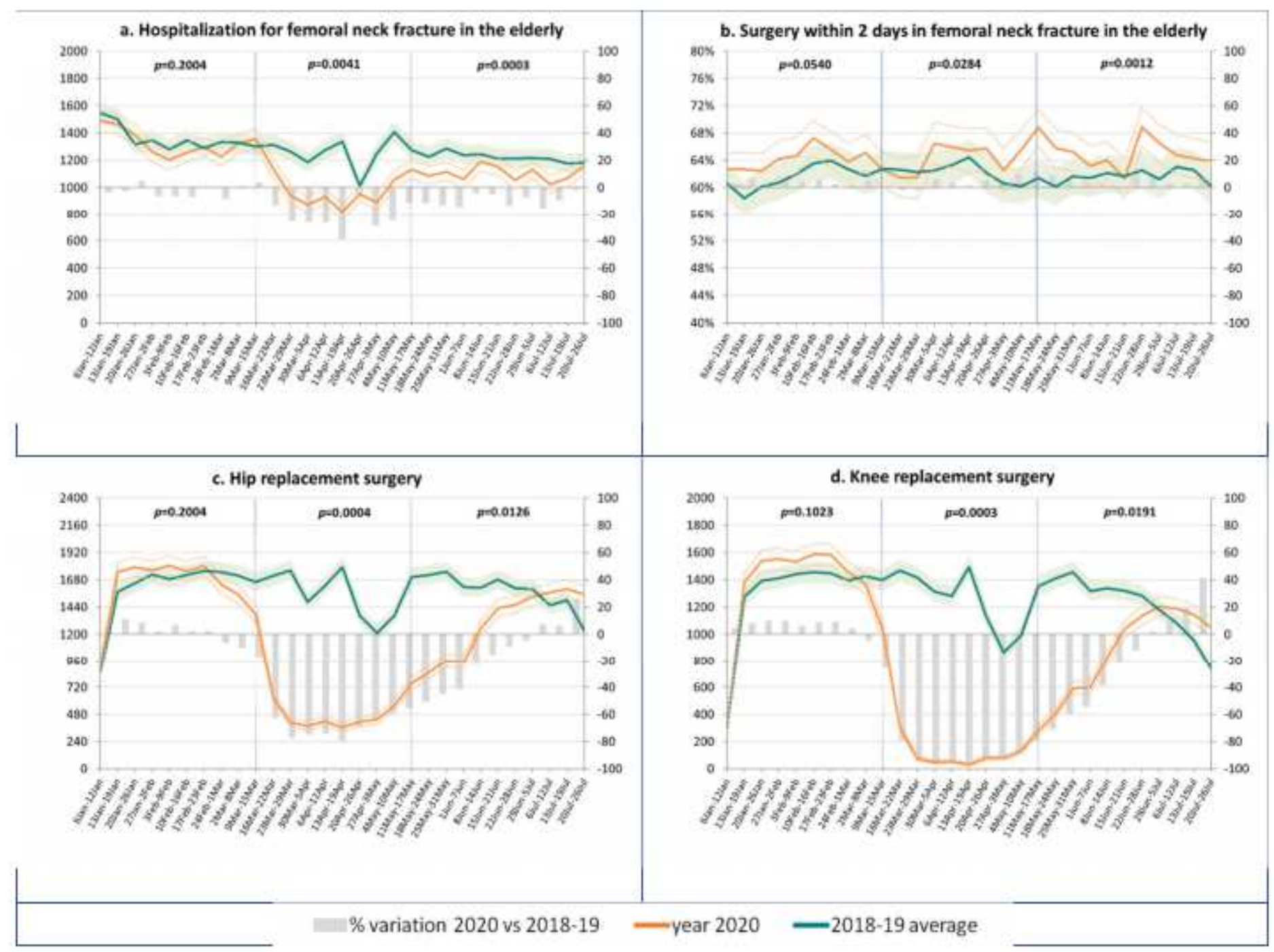

\section{Figure 3}

Impact of Covid-19 on orthopaedic hospital care in Italy: volumes and performance by sub-period* Weekly trend of indicators (left axis) with $95 \% \mathrm{Cl}$ and \% variations (right axis) - January-July 2020 vs. 2018-19 average. * pre-lockdown, lockdown (9/3-17/5), post-lockdown - p-values from the KruskallWallis test for comparisons within each subperiod

\section{Supplementary Files}

This is a list of supplementary files associated with this preprint. Click to download.

- OnlineResource1Listofindicators.pdf

- OnlineResource2FiguresS1S3byregion.pdf 